\title{
Un software educativo como una herramienta pedagógica en la mejora de las habilidades de lectoescritura utilizando el método ecléctico
}

\author{
Carlos Andrés Miranda Palma \\ Estudiante del doctorado en Tecnología Educativa de la \\ Facultad de Informática de la Universidad Autónoma de Querétaro (México) \\ cmiranda@correo.uady.mx
}

Rosa María Romero González

Profesora investigadora de tiempo completo de la Facultad de Informática de la Universidad Autónoma de Querétaro (México) y miembro del núcleo básico del doctorado en Tecnología Educativa

rossyrg04@yahoo.com.mx

\section{Extracto}

El método ecléctico es una técnica para apoyar a los estudiantes con problemas de lectoescritura. Este método se propone como estrategia de aprendizaje para el desarrollo de un software educativo que busca mejorar las habilidades lectoescritoras de los estudiantes con problemas de aprendizaje. Se menciona la situación que se pretende atender con este software educativo. Se analiza si el software educativo con estas características puede ser considerado como una herramienta pedagógica. Asimismo, se presentan las actividades, los instrumentos de medida y los indicadores del método ecléctico que se utilizarán para el desarrollo de las actividades de aprendizaje y para medir la utilidad del software educativo. Finalmente, se describe lo que se espera al utilizar el método ecléctico en la definición de las estrategias de aprendizaje y del estado actual del desarrollo del software educativo.

Palabras clave: estrategias de aprendizaje; habilidades lectoescritoras; herramienta pedagógica; método ecléctico; software educativo.

Fecha de entrada: 17-10-2018 / Fecha de aceptación: 09-01-2019

Cómo citar: Miranda Palma, C. A. y Romero González, R. M. ${ }^{\text {a }}$ (2019). Un software educativo como una herramienta pedagógica en la mejora de las habilidades de lectoescritura utilizando el método ecléctico. Tecnología, Ciencia y Educación, 13, 172-186. 


\title{
An educational software as a pedagogical tool in the improvement of reading and writing skills using the eclectic method
}

\author{
Carlos Andrés Miranda Palma \\ Rosa María Romero González
}

\section{Abstract}

The eclectic method is a method to support students with reading-writing problems. This method is proposed as a learning strategy for the development of educational software that seeks to improve the reading and writing skills of students with learning problems. The situation that is intended to be addressed with this educational software is mentioned. It is analyzed if the educational software with these characteristics can be considered as a pedagogical tool. Likewise, the activities, measurement instruments and indicators that will be used in the eclectic method for the development of learning activities and to measure the usefulness of educational software are presented. Finally, what is expected when using the eclectic method in the definition of learning strategies and the current state of educational software development is described.

Keywords: learning strategy; reading and writing skills; pedagogical tool; eclectic method; educational software.

Citation: Miranda Palma, C. A. y Romero González, R. M. ${ }^{\text {a }}$ (2019). An educational software as a pedagogical tool in the improvement of reading and writing skills using the eclectic method. Tecnología, Ciencia y Educación, 13, 172-186. 


\section{Sumario}

1. Introducción

2. Marco teórico

3. El software educativo como una herramienta pedagógica

4. Método ecléctico

5. Conclusiones

Referencias bibliográficas 


\section{Introducción}

Indiscutiblemente, la atención a estudiantes que presentan necesidades educativas especiales es una de las demandas prioritarias en el ámbito educativo. Para ello se han establecido instituciones de carácter público capaces de satisfacer los requerimientos que la sociedad presenta en dicha área. Existen zonas donde esta demanda es todavía más evidente, principalmente en los municipios de mayor población. Ante esta demanda, surge la iniciativa de establecer instituciones de carácter público que apoyen a estos estudiantes. Ejemplo de estos esfuerzos son las unidades de servicio de apoyo a la educación regular (USAER) establecidas en algunas escuelas primarias en el estado de Yucatán. De igual manera, en el estado de Querétaro se cuenta con la unidad de servicios para la educación básica (USEBEQ).

A través de actividades integradoras, estas unidades tienen como objetivo capacitar a los niños que van más rezagados en el aprendizaje de la lectoescritura para que consigan integrarse en el grupo con la finalidad de que se realicen como personas autónomas, posibilitando su inclusión y participación tanto en el grupo como en su comunidad.

Actualmente, en las USAER de la ciudad de Tizimín (Yucatán), los profesores se enfrentan a la situación de contar con algunos estudiantes por grupo que presentan problemas de lectoescritura. Estos niños no son canalizados a otras aulas para recibir una atención personalizada, sino que son atendidos dentro del mismo espacio de aprendizaje habitual y junto a sus demás compañeros. Debido a esta situación, los profesores se las ingenian para ser creativos y ofrecerles la atención adecuada. Además, el tipo y la cantidad de recursos materiales para llevar a cabo tales estrategias resultan ser limitados y los pocos materiales que existen suelen ser muy caros o son software propietario, por lo que los propios profesores los tienen que crear de forma manual. Asimismo, estas actividades o estrategias han de estar enfocadas para que las realicen todos los alumnos y no solo los niños con problemas de aprendizaje, ya que también se busca que los demás estudiantes mejoren sus habilidades, principalmente las cognitivas y las lectoras.

Los profesores se enfrentan a la
situación de contar con algunos
estudiantes por grupo que presentan
problemas de lectoescritura. Estos
niños no son canalizados a otras
aulas para recibir una atención
personalizada, sino que son
atendidos dentro del mismo espacio
de aprendizaje habitual y junto a
sus demás compañeros. Debido
a esta situación, los profesores se
las ingenian para ser creativos y
ofrecerles la atención adecuada

Los profesores se enfrentan a la situación de contar con algunos estudiantes por grupo que presentan problemas de lectoescritura. Estos niños no son canalizados a otras aulas para recibir una atención personalizada, sino que son atendidos dentro del mismo espacio de aprendizaje habitual y junto a sus demás compañeros. Debido a esta situación, los profesores se ofrecerles la atención adecuada 
Ante esta situación se propone el método ecléctico como estrategia de aprendizaje para el desarrollo de un software educativo de licencia libre que contribuya a la mejora de las habilidades lectoescritoras de los estudiantes de niveles de educación básica con problemas de aprendizaje. A través de este software, el estudiante podrá realizar actividades, de acuerdo con el método ecléctico, que le ayuden a mejorar sus habilidades de lectoescritura. La interacción con el usuario se producirá de forma amigable, ágil, divertida, dinámica y lúdica. Una vez concluido el desarrollo del software, se evaluará su utilidad en el desarrollo o en la mejora de las habilidades lectoescritoras de los estudiantes. El desarrollo de este software le permitirá al profesor del USAER contar con una herramienta más y podrá usarlo tanto de manera individual como grupal.

El software educativo que se desarrolla tendrá una historia (podría ser de espionaje o alguna otra que resulte interesante a los niños) y, dentro de este contexto, el niño tendrá que vencer retos (como descifrar un código o acertijos) para ir descubriendo nuevos escenarios en los que deberá realizar actividades que impliquen la comprensión lectora, entre otras actividades de lectoescritura. Una vez que se consiga realizar la actividad propuesta, el niño avanzará a las siguientes etapas del juego. Se proponen dos videojuegos con sus respectivas estrategias de aprendizaje (método ecléctico) y retos de inicio de cada etapa.

El desarrollo de un software con estas características puede ser un medio a través del que se logre establecer un contacto entre la tecnología y el área de educación especial. Además, los niños siempre se muestran muy interesados en aprender a través de la tecnología.

Hoy en día, la tecnología ha revolucionado de manera vertiginosa nuestras vidas (ha entrado a formar parte de nuestra vida diaria y hacemos uso de sus aplicaciones en nuestras labores cotidianas). Particularmente, la aplicación tecnológica en el área de educación especial con niños que tienen problemas de aprendizaje ya tiene lugar en algunos países; sin embargo, en México todavía dista de ser algo tangible. El propósito de esta investigación es el desarrollo de un software educativo que utilice el método ecléctico como estrategia de aprendizaje y que pueda ser considerado como una herramienta pedagógica que apoye en la mejora de las habilidades lectoescritoras de los estudiantes del primer y/o segundo curso de primaria. 


\section{Marco teórico}

Según Ferrés y Marquès (1996), un software educativo es un programa para ordenador, creado con el fin de ser utilizado como medio didáctico, que pretende imitar la labor tutorial que realizan los profesores y que presenta modelos de representación del conocimiento en consonancia con los procesos cognitivos que desarrollan los alumnos. Por lo tanto, está centrado en el proceso de enseñanza-aprendizaje y pretende atender las necesidades del estudiantado en función de los programas educativos.

El software educativo surge por la necesidad de incorporar nuevas estrategias de enseñanza y con el fin de agilizar y facilitar este proceso en las aulas. Se definen como softwares educativos los programas informáticos que son realizados con la finalidad de ser utilizados como facilitadores del proceso de enseñanza y, consecuentemente, de aprendizaje, y que cuentan con algunas características particulares, tales como la facilidad de uso, la interactividad y la posibilidad de personalización de la velocidad del aprendizaje (Cataldi, 2000).

Sánchez (2000) define el software educativo como cualquier programa informático cuyas características estructurales y funcionales le permiten servir de apoyo a la enseñanza, al aprendizaje y a la administración educacional. Las expresiones «software educativo», "programas educacionales» y «programas didácticos» se usan como sinónimos para designar genéricamente todo tipo de programas informáticos creados con la finalidad específica de ser utilizados como medios didácticos.

En general, existen una gran variedad de definiciones para el término «software educativo", sin embargo, la mayoría de ellas comparten una serie de rasgos comunes que son los que deben caracterizar a un software para ser considerado educativo: finalidad didáctica, intencionalidad pedagógica, apoyo curricular, material pedagógico y medio didáctico (Fallas y Chavarría, 2010).

Finalmente, Pérez y Gardey (2016) mencionan que algunos softwares educativos son diseñados como apoyo al docente. De esta manera, el profesor recurre al software para ofrecer sus lecciones o para reforzar una clase. Otros tipos de softwares educativos, en cambio, se orientan directamente al alumno, ofreciéndole un entorno en el cual puede aprender por su propia cuenta. Esto último es lo que se pretende con el software educativo, ya que no se busca ofrecer lecciones sobre temas, sino que el alumno aprenda o refuerce sus conocimientos dentro de un entorno de aprendizaje.

El software que se va a desarrollar cumplirá con las características y las finalidades de un software educativo y también brindará un entorno donde los estudiantes de primer y/o segundo curso de primaria mejoren y/o refuercen por su propia cuenta sus habilidades lectoescritoras. 
Los métodos más comunes que se utilizan para mejorar las habilidades de los niños con problemas de aprendizaje en el área de lectoescritura los menciona Salavarrieta (2015) y son los siguientes:

- Método silábico.

- Método alfabético o deletreo.

- Método global.

- Método ecléctico.

- Método Negret.

Posteriormente se describirá con detalle el método ecléctico, que es el método que se propone como estrategia de aprendizaje para el software educativo.

Para el desarrollo de este software educativo se utilizará la investigación basada en diseño (IBD), bajo un paradigma cuantitativo, y se ha dividido en las siguientes etapas:

- Definición del problema.

- Diseño y desarrollo.

- Implementación.

- Validación.

- Evaluación.

La IBD ha sido desarrollada dentro de las ciencias del aprendizaje (learning sciences) y se nutre de un amplio campo multidisciplinar que incluye la antropología, la psicología educativa, la sociología, la neurociencia, así como las didácticas específicas, entre otras (Confrey, 2006; Sawyer, 2006). Esta metodología tiene como objetivo analizar el aprendizaje basado en contexto mediante el diseño y el estudio sistemático de formas particulares de aprendizaje, estrategias y herramientas de enseñanza, de una forma sensible a la naturaleza sistémica del aprendizaje, la enseñanza y la evaluación. Todo ello la convierte en un paradigma metodológico potente en la investigación del aprendizaje y la enseñanza (Molina, Castro, Molina y Castro, 2011).

\section{El software educativo como una herramienta pedagógica}

Flórez (1994) menciona que la actividad creadora no es programable ni enseñable y que la sola actividad neuronal computable no genera pensamiento, ni imaginación creadora, por lo que el software educativo puede ser una herramienta que ayude a despertar o a in- 
centivar esa actividad creadora que los niños tienen. Primeramente, el software educativo puede ser una herramienta pedagógica; todo dependerá de la forma en que sea diseñado y utilizado. Flórez menciona que la misión y el eje teórico de la pedagogía es la formación humana. Y es ahí donde el software educativo -con el diseño de las actividades adecuadas (de acuerdo a la estrategia de aprendizaje), y que estas se realicen de una manera amigable, ágil, divertida, dinámica y lúdica- permita al usuario usar su imaginación y creatividad. De esta forma, el software educativo será una herramienta que cumpla con la formación humana e incentive la creatividad. Ambas características son importantes y deben ir de la mano, ya que las estrategias de aprendizaje permiten definir metas, buscar objetivos y desarrollar ciertas habilidades que contribuyan a la formación del usuario, pero estas estrategias no deben ser implementadas de una manera formal o aburrida, sino a través de actividades divertidas, amenas y lúdicas que permitan al usuario, y en particular a los niños, mejorar sus habilidades o desarrollar una parte formativa sin que estos se den cuenta o sientan que están repasando o practicando; más bien deben pensar que están jugando o entreteniéndose, y, de esa manera, tal y como ocurre en la vida real, al estar jugando o realizando ciertas actividades, aprenderán sin darse cuenta.

Flórez (1994) propone el cumplimento de tres condiciones para validar el concepto de «formación» para la pedagogía. Para la condición antropológica, cuando el usuario utiliza el tipo de software educativo que proponemos, pondría a prueba sus conocimientos previos y, al ir avanzando, iría desarrollando nuevas habilidades o conocimientos, cumpliendo de esa manera con esta condición. Con este tipo de software educativo, cuando el usuario supere los retos o las actividades de aprendizaje, avanzará a otros niveles de aprendizaje; de esta manera, el usuario sentirá que está avanzando en sus conocimientos y habilidades, y, con ello, se cumplirá la condición teleológica que propone Flórez. Para la condición metodológica, este tipo de software educativo seguirá una metodología o estrategia de aprendizaje (método ecléctico) que en todo momento permitirá cuestionar si las actividades o estrategias cumplen con su función y, si no cumplen, reorientarlas. Al cumplir con las tres condiciones, este tipo de software educativo cumpliría con el concepto de «formación» que describe Flórez.

De lo anterior se deduce que el software educativo, y, en particular, el que se describe en este trabajo, puede ser una herramienta pedagógica que ayudaría a mejorar las habilidades lectoescritoras de los estudiantes.

No sirve exclusivamente con que el software educativo cuente con los elementos anteriores, sino que también es importante la forma en que se utilice en clase, tal y como comenta Quintanilla (2005). Este autor menciona que la técnica influye en todos los aspectos de la vida humana y, sobre todo, en las innovaciones, ya que un cambio en la técnica puede arrojarnos mejores resultados. El software educativo en sí mismo puede ser una buena herramienta, pero el uso o la técnica que el profesor le dé en el aula harán que los resultados sean unos u otros. El software puede presentar la mejor estrategia de aprendizaje, las mejores actividades lúdicas y entretenidas, la mejor finalidad, etc., y probablemente se ob- 
tendrán buenos resultados, pero si el profesor emplea una buena técnica para aprovechar todas las bondades y los beneficios del software, entonces se podrían conseguir resultados más eficaces y en mucho menos tiempo.

Precisamente ahí es donde está el reto de estas herramientas, ya que el profesor que decida utilizar este tipo de software educativo tiene que tener un conocimiento operacional y estructural (know how), y conocer la naturaleza del software y las relaciones entre el conocimiento tecnológico y pedagógico del mismo (Quintanilla, 2005).

\section{Método ecléctico}

Bartra (2007) comenta que Tattersal creía que los mecanismos periféricos del habla no fueron una adaptación, sino una mutación que ocurrió varios cientos de miles de años antes de que quedaran circunscritos por la función de articular sonidos. Asimismo, considera que nuestras capacidades cognitivas actuales fueron también una transformación ocurrida hace unos 100.000 o 150.000 años que no fue aprovechada hasta hace 60.000 o 70.000 años, cuando ocurrió una innovación cultural que activó en algunos humanos arcaicos el potencial para realizar los procesos cognitivos simbólicos que residían en el cerebro sin ser empleados. Según Tattersall, el detonador de este proceso cultural fue la invención del lenguaje. Se menciona que la habilidad lingüística tenía ya un cableado neuronal inscrito en el cerebro y que solo faltaba el estímulo externo para ponerlo a funcionar. Ese disparador pudo haber sido algo tan sencillo como una invención realizada por un grupo de niños durante sus juegos. Una vez que se hizo esta maravillosa invención, el conjunto de la sociedad debió de adoptarla y difundirla a otros grupos. Bartra menciona que se debe aceptar que la transformación neuronal comenzó a tener consecuencias desde el momento en que un subgrupo de homínidos tuvo que enfrentarse a retos que superaban los recursos normalmente usados y que no fue producto del azar o de un juego de niños.

Teniendo en cuenta lo anterior, a través del tiempo se han definido estrategias de aprendizaje para mejorar las habilidades lectoescritoras de los estudiantes. El objetivo es buscar que los alumnos, a través de retos o actividades, vayan mejorando sus habilidades cognitivas, empleando para ello estímulos externos e internos y estrategias innovadoras que buscan detonar ese potencial que cada uno tiene y, de este modo, mejorar nuestras habilidades. A continuación, mencionaremos las características del método ecléctico, estrategia de aprendizaje para el software que se propone.

El método ecléctico es aquel que se forma de lo más significativo y valioso de los demás métodos:

- Del método silábico toma el análisis de palabras hasta llegar a la sílaba. Emplea el silabario como estímulo para lograr el perfeccionamiento. 
- Del método alfabético toma el ordenamiento de las letras, las imágenes para recordar las letras, y trabaja las mayúsculas y las minúsculas al mismo tiempo.

- Del método global de la primera etapa toma el reconocimiento de palabras por contexto; de la segunda etapa, los diversos ejercicios de escritura y dictados; de la tercera etapa, el reconocimiento de palabras o partes de la palabra en otra palabra; y de la cuarta etapa, la lectura comprensiva y la escritura en letra script y cursiva.

- De las palabras normales toma ejercicios de pronunciación y articulación, imágenes y representación de elementos, combinación de palabras-sílabas y letras.

Como se mencionó anteriormente, el software educativo que se propone seguirá el método ecléctico y, para ello, es necesario describir las actividades, los instrumentos de medida y los indicadores que servirán para definir las estrategias de aprendizaje y los indicadores que verificarán la utilidad del software. A continuación, mencionaremos cada uno de ellos y los que se proponen utilizar para este software.

En primer lugar, comencemos con las actividades del método ecléctico que se utilizarían como estrategias de aprendizaje. García y Escrig (citados en Cristóbal, 2013) se apoyan en esta metodología, y algunas de las actividades y finalidades que han planteado para este método se pueden observar en el cuadro 1 (en la columna de la izquierda se presentan las actividades y en la columna de la derecha la finalidad pedagógica de cada una de las actividades propuestas).

Cuadro 1. Actividades y finalidades pedagógicas del método ecléctico

Actividad

Portada de la letra en mayúscula y minúscula con el dibujo y la palabra correspondiente.

Presentación de la letra que se va a trabajar en mayúscula y minúscula.
Finalidad pedagógica
Presentación de la letra que hay que trabajar para que el alumno asocie dicha letra con un dibujo muy conocido para él.

Interiorización de la letra, trabajando de diferentes maneras: repaso con el dedo, realización de la letra en el aire, pegando plastilina, bolas de papel de seda o trozos de periódico, etc.

Discriminación e interiorización visual de la grafía de la letra trabajada.

Discriminación, asociación y memorización de las palabras presentadas con sus dibujos.
Presentación de ocho palabras con su dibujo para leerlas. 
Unión de las palabras con su dibujo (las palabras presentadas en la actividad anterior y su respectivo dibujo aparecen mezclados).

Dado el dibujo y la palabra escrita sin la letra trabajada, escribirla.

Caligrafía de la letra trabajada en mayúscula y minúscula con una muestra, con letra punteada para repasar y, finalmente, sin ningún tipo de ayuda.

Actividad para repasar palabras punteadas (las trabajadas anteriormente) y luego escribirlas sin ayuda.

Dibujar palabras con el sonido de la letra trabajada.

Copiar y clasificar en una tabla las palabras según la letra por la que empiecen.

Dibujar las palabras anteriores en dos nubes según empiecen.

A partir de una frase sencilla con palabras de las actividades anteriores, dibujarla.

Búsqueda visual de las letras trabajadas entre las letras del abecedario y redondearlas en diferentes colores.

Ordenar las palabras mezcladas de las frases anteriores.

Con el apoyo visual del dibujo, leer y contestar, de entre dos palabras muy parecidas, cuál es la correcta.
Reconocimiento visual de las palabras presentadas asociándolas a su dibujo correspondiente.

Reconocimiento de la letra que falta y producción de esta, trabajando así la ruta fonológica.

Escritura de la letra con correcta direccionalidad, cogiendo de manera adecuada el lápiz y trabajando la habilidad óculo-manual.

Escritura de la palabra con correcta direccionalidad, cogiendo de manera adecuada el lápiz y trabajando la habilidad óculo-manual.

Discriminación auditiva de palabras que contengan la grafía trabajada en cualquier posición de la palabra.

Discriminación visual y auditiva de la grafía contenida en las palabras trabajadas y que ya son conocidas por el alumno.

Discriminación visual y auditiva de palabras que contengan dos grafías trabajadas, cogiendo correctamente el lápiz.

Discriminación visual y auditiva; comprensión lectora y motricidad fina.

Discriminación visual de las grafías trabajadas.

Reconocer, comprender y escribir las palabras formando una oración con sentido.

Discriminación visual y auditiva, comprensión, asociación y escritura de la palabra correcta. 
De todas estas actividades, las que son viables para ser desarrolladas en el software educativo son las siguientes:

- Presentación de la letra que se va a trabajar en mayúscula y minúscula.

- Pintar y picar la letra en mayúscula y minúscula.

- Presentación de ocho palabras con su dibujo para leerlas.

- Unión de las palabras con su dibujo (las palabras presentadas en la actividad anterior y su respectivo dibujo aparecen mezclados).

- Dado el dibujo y la palabra escrita sin la letra trabajada, escribirla.

- Dibujar palabras con el sonido de la letra trabajada.

- Copiar y clasificar en una tabla las palabras según la letra por la que empiecen.

- Dinujar las palabras anteriores en dos nubes según empiecen.

- A partir de una frase sencilla con palabras de las actividades anteriores, dibujarla.

- Ordenar las palabras mezcladas de las frases anteriores.

- Con el apoyo visual del dibujo, leer y contestar, de entre dos palabras muy parecidas, cuál es la correcta.

Una vez definidas las actividades de aprendizaje es necesario establecer cuáles serían los instrumentos de medida para poder evaluar el avance en el desarrollo de las habilidades lectoescritoras de los niños. Revisando la literatura se encontró que se han diseñado algunos instrumentos que miden este avance. Estos instrumentos permiten analizar los resultados para ver si el método de lectoescritura repercute en el aprendizaje. A continuación, mencionamos estas pruebas.

Para Vallés y Vallés (citados en Cristóbal, 2013), las pruebas psicopedagógicas más actuales para evaluar la lectura son:

- PROLEC (procesos lectores/nivel de educación primaria: 10 subtest). Esta prueba evalúa las rutas fonológica, léxica, sintáctica y semántica.

- PRUEBAS ACL (evaluación de la comprensión lectora). Evalúa del nivel $1 .^{\circ}$ al $6 .^{\circ}$ de educación primaria.

- ECL (evaluación de la comprensión lectora). Evalúa a los niños que tienen entre 7 y 16 años.

Para Cuetos (citado en Cristóbal, 2013), una prueba psicopedagógica para evaluar la escritura es PROESC, que evalúa los procesos de escritura para niños entre 8 y 16 años. 
Este instrumento cuenta con las siguientes actividades: dictado de palabras, de pseudopalabras y de frases, y escritura de un cuento y de una redacción.

De igual forma, se pretende que el software retroalimente al usuario al proporcionarle cuáles han sido sus aciertos y logros en el desarrollo de las actividades o de los retos. De esta manera se podrá evaluar el avance de los niños, ya que es posible comparar los resultados obtenidos con los que se hayan logrado en interacciones anteriores.

Asimismo, para verificar si existe una diferencia o mejora en las habilidades lectoescritoras de los estudiantes que utilizaron el software y de los que no, se les podría aplicar, a ambos grupos de niños, alguna de las pruebas mencionadas anteriormente y, con ello, comparar los avances de los estudiantes.

Finalmente, para verificar la utilidad del método usando el software, se necesita definir una serie de indicadores para comparar los avances en las habilidades lectoescritoras entre los estudiantes que aprenden con un método ecléctico y los que no, por lo que se propone utilizar los indicadores que Ortega (2009) describe en su trabajo doctoral.

De estos indicadores se han seleccionado aquellos que son actividades viables para ser implementados en los juegos o en las actividades del software educativo. Estos indicadores son discriminación auditiva, segmentación de palabras, errores de omisión en la copia, errores de adición en la copia, separación de palabras en la copia, formación de palabras con las sílabas dadas, discriminación visual-gráfica (seleccionar la sílaba), discriminación visual-gráfica (completar la palabra con la sílaba correspondiente), asociación imagen-palabra, errores de sustitución en el dictado, errores de omisión en el dictado, errores de adicción en el dictado, escritura de la palabra correspondiente a la imagen dada, escritura de palabras con «pr» o «pl», verificación de frases correctas con las palabras dadas, empleo de la mayúscula, etc. Estos indicadores permitirán evaluar las siguientes actividades: dictado de palabras y de frases, la comprensión lectora, dibujar lo que se le indica, seleccionar la ilustración correcta y la redacción.

Con estos indicadores se podrá elaborar una prueba o cuestionario que se aplicará tanto a los estudiantes que utilicen el software como a los que no lo hayan utilizado y con los resultados que se obtengan se podría verificar si existe una diferencia importante en las habilidades lectoescritoras entre los niños que usan el software y los que no, y con ello probar la utilidad del mismo.
Con los resultados que se obtengan se podría verificar si existe una diferencia importante en las habilidades lectoescritoras entre los niños que usan el software y los que no, y con ello probar la utilidad del mismo 


\section{Conclusiones}

Un software educativo que utilice el método ecléctico para contribuir a mejorar las habilidades lectoescritoras de los estudiantes con problemas de aprendizaje de lectoescritura y que permita retroalimentar y observar el avance y los logros al realizar las actividades de aprendizaje es una aportación importante para acercar la tecnología a la educación especial. Este software educativo se conformará con dos juegos integrados. Para cada juego se definirán estrategias de aprendizaje siguiendo el método ecléctico, y estas actividades permitirán al estudiante alcanzar un objetivo cuantificable de manera divertida, ágil y amigable. Para lograrlo, el alumno necesitará vencer los retos o acertijos que se le presenten y realizar de manera correcta las actividades de aprendizaje.

Como se mencionó anteriormente, el método ecléctico es aquel que se forma de lo más significativo y valioso de los demás métodos que se utilizan para mejorar las habilidades lectoescritoras de los niños. Al utilizarlo en un software educativo como estrategia de aprendizaje para las actividades de entretenimiento y de mejora de las habilidades lectoescritoras, puede ser una aportación innovadora, sobre todo en el área de la educación especial, ya que, como se mencionó, los profesores de esta área se las ingenian para poder crear actividades y materiales (de manera manual) con los que apoyar a estos estudiantes y que, en estas actividades, todos los estudiantes del aula se vean involucrados.

Actualmente, el software educativo se encuentra en la etapa de diseño y desarrollo. Se han definido tres personajes con los cuales los estudiantes se podrían identificar y a los que ayudarán a vencer los retos que se les vayan presentando. Asimismo, se han definido las historias y una parte de las actividades de aprendizaje (siguiendo el método ecléctico) de cada uno de los juegos que conformarán el software

Un software educativo con estas características puede ser una herramienta pedagógica y, también, una herramienta que apoye el desarrollo o la mejora de las habilidades de los estudiantes que presentan dificultades. Esta es un área que no se ha explorado del todo y que ofrece oportunidades de investigación educativo. Un software educativo con estas características puede ser una herramienta pedagógica y, también, una herramienta que apoye el desarrollo o la mejora de las habilidades de los estudiantes que presentan dificultades, lo que diversifica aún más los beneficios y los usos que se le pueden dar a esta herramienta tecnológica. Si además se utiliza con una buena técnica, los resultados pueden ser muy productivos. Esta es un área que no se ha explorado del todo y que ofrece oportunidades de investigación. 


\section{Referencias bibliográficas}

Bartra, R. (2007). Antropología del cerebro: la conciencia y los sistemas simbólicos. México: Fondo de Cultura Económica (FCE).

Cataldi, Z. (2000). Una metodología para el diseño, desarrollo y evaluación de software educativo (Tesis de maestría en informática/Versión resumida). Facultad de Informática UNLP. Recuperado de <http:// sedici.unlp.edu.ar/handle/10915/4055> (consultado el 20 de julio de 2018).

Confrey, J. (2006). The evolution of design studies as methodology. En R. K. Sawyer (Ed.), The Cambridge Handbook of the Learning Sciences (pp. 135-152). New York: Cambridge University Press.

Cristóbal Muñoz, S. (2013). La metodología de lectoescritura en educación infantil y su influencia en el aprendizaje lectoescritor de los alumnos. Universidad de Valladolid. Recuperado de <http://uvadoc.uva. es/handle/10324/3204> (consultado el 11 de septiembre de 2018).

Fallas Monge, J. J. y Chavarría Molina, J. (abril 2010). Validación de software educativo. VII Festival Internacional de Matemática. Instituto Tecnológico de Costa Rica.

Ferrés Prats, J. y Marquès Graells, P. (Coords.). (1996). Comunicación educativa y nuevas tecnologías. Barcelona: Editorial Praxis.

Flórez Ochoa, R. (1994). Hacia una pedagogía del conocimiento. Chile: Editorial McGraw-Hill.

Molina, M., Castro, E., Molina, J. L. y Castro, E. (2011). Un acercamiento a la investigación de diseño a través de los experimentos de ense- ñanza. Investigación Didáctica, Enseñanza de las Ciencias, 29(1), 75-88. Recuperado de <www.raco.cat/index.php/Ensenanza/arti cle/viewFile/243824/353427> (consultado el 15 de septiembre de 2018).

Ortega Sánchez, R. M. ${ }^{a}$. (2009). Estudio y análisis del método ecléctico de lectoescritura en las escuelas de la SAFA (Tesis de doctorado). Departamento de Didáctica y Organización Escolar, Universidad de Granada. Recuperado de <https://hera.ugr. es/tesisugr/17899151.pdf> (consultado el 13 de septiembre de 2018).

Pérez Porto, J. y Gardey, A. (2016). Definición de software educativo. Definición. de. Recuperado de <https://definicion.de/ software-educativo/> (consultado el 20 de julio de 2018).

Quintanilla, M. Á. (2005). Tecnología: un enfoque filosófico y otros ensayos de filosofía de la tecnología. México: Fondo de Cultura Económica (FCE).

Salavarrieta Tunjo, F. M. (2015). Aprendiendo a leer: cartilla de lectura. Práctica Profesional. Corporación Universitaria Minuto de Dios. Facultad de Educación.

Sánchez, J. (2000). Nuevas tecnologías de la información y comunicación para la construcción del aprendizaje. Santiago de Chile: Universidad de Chile.

Sawyer, R. K. (2006). The new science of learning. En R. K. Sawyer (Ed.), The Cambridge Handbook of the Learning Sciences (pp. 1-18). New York: Cambridge University Press. 